\title{
Popular Content Distribution in CR-VANETs with Joint Spectrum Sensing and Channel Access using Coalitional Games
}

\author{
Tianyu Wang ${ }^{1}$, Lingyang Song ${ }^{1, *}$, Zhu Han $^{2}$ \\ ${ }^{1}$ School of Electrical Engineering and Computer Science, Peking University, Beijing, China. \\ ${ }^{2}$ Electrical and Computer Engineering Department, University of Houston, Houston, USA.
}

Abstract

Driven by both safety concerns and commercial interests, popular content distribution (PCD), as one of the key services offered by vehicular networks, has recently received considerable attention. In this paper, we address the PCD problem in highway scenarios, in which a popular file is distributed to a group of onboard units (OBUs) driving through an area of interest (AoI). Due to high speeds of vehicles and deep fading of vehicle-to-roadside (V2R) channels, the OBUs may not finish downloading the entire file. Consequently, a peer-to-peer $(\mathrm{p} 2 \mathrm{p})$ network should be constructed among the OBUs for completing the file delivery process. Here, we apply the cognitive radio technique for vehicle-to-vehicle communications and propose a cooperative approach based on coalition formation games, which jointly considers the spectrum sensing and channel access performance. Simulation results show that our approach presents a considerable performance improvement compared with the non-cooperative case.

Received on 01 October 2013; accepted on 17 March 2014; published on 14 July 2014

Keywords: VANET, cognitive radio, coalitional games

Copyright (C) 2014 Tianyu Wang et al., licensed to ICST. This is an open access article distributed under the terms of the Creative Commons Attribution license (http://creativecommons.org/licenses/by/3.0/), which permits unlimited use, distribution and reproduction in any medium so long as the original work is properly cited.

doi:10.4108/ws.1.2.e4

\section{Introduction}

Vehicular ad hoc networks (VANETs) have been envisioned to provide increased convenience and efficiency to drivers, with numerous applications ranging from traffic safety, traffic efficiency to infotainment [1, 2]. One particular type of service, popular content distribution (PCD) has attracted a lot of attentions recently, in which multimedia contents are distributed from roadside units (RSUs) to on-board units (OBUs) driving through an area of interest (AoI) [3-5]. Examples of PCD may include: a local hotel periodically broadcasts multimedia advertisements to the vehicles entering the city on suburban highway; and a traffic authority delivers real-time traffic information ahead, or disseminates an update version of local GPS map [3].

Unlike downloading services on the Internet or their direct extensions in VANETs where various vehicles are interested in downloading different files [6, 7], the PCD is different in the following characteristics:

1. The content for dissemination is a single large file, such as an emergency video.

2. The only interested users are the OBUs driving through an AoI.

*Corresponding author. lingyang.song@pku.edu.cn
3. The OBUs are moving at high speeds with unstable network topology.

4. The wireless links are unreliable and may suffer interferences from each other.

Due to the high speeds of vehicles and the large file size of popular content, the OBUs may fail to download the entire popular file within the limited time for vehicleto-roadside (V2R) communications, and each OBU receives only a portion depending on the location and speed of passing the AoI. However, in most cases, the OBUs as whole has already obtained all the segments of the popular file when they sequentially pass the AoI. For completing the file delivery process, a peer-to-peer (p2p) network can be constructed among the OBUs, in which popular segments can be transmitted by vehicle-to-vehicle (V2V) communications. Considering the pressing demand of spectrum, the cognitive radio technique can be applied in V2V communication and the p2p network therefore turns out to be a cognitive radio vehicular ad hoc network (CR-VANET), where the OBUs as secondary users (SUs) sense the licensed spectrum for primary users (PUs) and access the PU channels at vacant time. Actually, in the proposed highway scenario where most portions are in rural areas, the spectrum is generally quite clean 
and cognitive radio is always a suitable and efficient technique.

Peer-to-peer (p2p) networks, which are distributed systems without any hierarchical organization or centralized control, have become immensely popular on the Internet in both application field and academic field [8]. Based on the existence of prior knowledge of network topology, p2p networks can be classified into structured $\mathrm{P} 2 \mathrm{P}$ systems and unstructured $\mathrm{P} 2 \mathrm{P}$ systems [8]. In our scenario, the network topology is unknown due to the randomness of OBUs. Thus, the $\mathrm{p} 2 \mathrm{p}$ system in this paper is an unstructured p2p system as the BitTorrent and eDonkey. However, those $p 2 p$ techniques used on the Internet should be carefully inspected before applying in our problem. First, the network topology is not only unknown but also ever-changing due to the mobility of OBUs. Second, the wireless links are very unreliable and may even interfere each other. Thus, the existing methods for Internet services can be inefficient and novel approaches should be proposed. Moreover, by introducing the cognitive radio technique, the performance of spectrum sensing and the throughput of channel access should be jointly considered in order to maximize the efficiency of the network, which also increases the complexity of networking.

In this paper, we propose a distributed approach based on coalition formation games to address the PCD problem in CR-VANETs. Coalitional game as a game theory model, in which players adopt cooperative strategies to form a coalition for improving individual profits, has recently been for modeling the cooperative behaviors of communication nodes. In [9], the cooperation between RSUs for V2R communication has been modeled as a coalition formation game with transferable utility, and the RSUs have been partitioned into many coalitions each of which applies a cooperative protocol to maximize their profits. In [10], coalitional games in partition form have been used for modeling the cooperation between SUs in cognitive radio networks, and the SUs in each coalition jointly sense the spectrum and coordinately access the PU channel for improving the total performance. In this paper, the considered p2p network not only has an ever-changing topology but also is strongly affected by the initial content distribution and the corresponding content request, which is different from the RSUs network in [9] or the SUs network in [10]. We propose a coalition formation game with non-transferable utility for modeling the cooperation between OBUs, which jointly considers the performance of cooperative sensing and the throughput of channel access.

The rest of this paper is organized as follows. Section II provides the system model and the non-cooperative spectrum sensing and access. In Section III, we present a coalition formation game with non-transferable

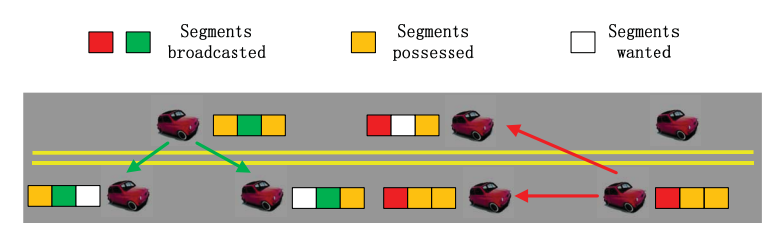

Figure 1. System model of the popular content distribution problem in cognitive radio ad hoc vehicular networks.

utility for modeling the cooperative manner, while in Section IV the proposed approach based on a coalition formation algorithm is presented for the entire PCD problem in CR-VANETs. In Section V simulation results and analysis are presented, and in Section VI we conclude the paper.

\section{System Model}

\subsection{Network Model}

Consider a CR-VANET with $N$ OBUs (SUs) engaged in the sensing of $K$ PU channels in order to access the spectrum and transmit data for PCD applications. A popular file, which is equally divided into $M$ segments, has been requested by all the OBUs. Let $\mathcal{N}, \mathcal{K}$ and $\mathcal{M}$ denote the set of OBUs, the set of PU channels and the set of segments, respectively. Due to the fast speeds of OBUs and deep fading of V2R channels, each OBU in $\mathcal{N}$ only receives a part of all the $M$ segments directly from RSUs, and the rest are gradually achieved by $\mathrm{p} 2 \mathrm{p}$ transmissions using $\mathrm{V} 2 \mathrm{~V}$ communications. Let $\mathcal{M}_{i}$ denote the set of segments possessed by OBU $i$, the initial elements of which are randomly distributed in $\mathcal{M}$.

We suppose the OBUs of the $\mathrm{p} 2 \mathrm{p}$ network periodically sense and access the PU channels, cooperatively or individually. In each cycle, or we say time slot, any OBU $i \in \mathcal{N}$ can decide to access one of the empty PU channels, the set of which is denoted by $\mathcal{K}_{i}$, or not to access. If OBU $i$ decides to access PU channel $k_{i} \in \mathcal{K}_{i}$, then OBU $i$ broadcasts one and only one segment $m_{i} \in$ $\mathcal{M}_{i}$ in PU channel $k_{i}$ for the current slot. We suppose the data traffic of PU channels can be modeled as $K$ independent Poisson processes with the same arriving rate $\lambda$ per time slot. The PU channel is empty only when no package of PU arrives at the current slot.

\subsection{Channel Model}

For any OBU $i$ broadcasting at any PU channel $k_{i}$, we suppose only the "neighbors" (the OBUs with a line of sight to OBU $i$ ), denoted by $\mathcal{N}_{i}$, can receive the signal [12]. For a given group of OBUs $S$ broadcasting in the current slot, we suppose only the non-interfered neighbors of any OBU $i \in S$, the set of which is denoted by $\mathcal{N}_{i}^{*}$, have the possibility of successfully achieving a 
segment. Thus, we have

$$
\begin{aligned}
& \mathcal{N}_{i}^{*}=\left\{j \in \mathcal{N}_{i} \mid \forall l \in\left(\mathcal{N}_{j} \backslash\{i\}\right) \cap S, k_{l} \neq k_{i},\right. \\
& \text { and } \left.k_{j} \neq k_{i} \text {, if } j \in S\right\} \text {. }
\end{aligned}
$$

The set of non-interfered neighbors of any OBU $i$, denoted by $\mathcal{N}_{i}^{*}$, represents the OBUs that are within the range of OBU $i$ but not within the range of any other OBU broadcasting via channel $k_{i}$.

We suppose the possibility of successful delivery between any two non-interfered OBUs is proportional to the corresponding V2V channel capacity. Here, we only consider the pathloss without any small-scale fading, and the capacity of PU channel $k$ between OBU $i$ and $\mathrm{OBU} j$ is given by

$$
c_{i, j}^{k}= \begin{cases}W \log _{2}\left(1+\alpha d_{i, j}^{-n}\right), & \text { LOS exits, } \\ 0, & \text { otherwise, }\end{cases}
$$

where $d_{i, j}$ is the distance between OBU $i$ and OBU $j, n$ is the pathloss exponent, $\alpha$ is a scale factor representing signal-to-noise ratio (SNR) at transmitters, and $W$ is the bandwidth of any PU channel. Thus, the possibility of successful delivery between OBU $i$ and OBU $j$ through PU channel $k$ is given by

$$
p_{i, j}^{k}= \begin{cases}0, & c_{i, j}^{k}<c, \\ \frac{c_{i, j}^{k}-c}{4 c}, & c \leq c_{i, j}^{k} \leq 5 c, \\ 1, & c_{i, j}^{k}>5 c,\end{cases}
$$

where $c$ is the size of any segment in $\mathcal{M}$. In (3), we assume the probability of success is zero if the capacity is less than the data rate, i.e., $c_{i, j}^{k}<c$, and the probability of success is 1 if the capacity is five times greater than the data rate, i.e., $c_{i, j}^{k}>5 c$. If $c<c_{i, j}^{k}<5 c$, we assume the probability of success is proportional to the capacity.

\subsection{Mobility Model}

The mobility model we use is similar to the Freeway Mobility Model (FMM) proposed in [13], which is well accepted for modeling the traffic in highway scenarios. In FFM, the simulation area includes many multiple lane freeways without intersections. At the beginning of the simulation, the vehicles are randomly placed in the lanes, and move at history-based speeds. The vehicles randomly accelerate or decelerate with the security distance $d_{s}>0$ maintained between two subsequent vehicles in a lane and no change of lanes is allowed.

In our scenario, the map has been simplified to a oneway traffic road with double lanes as shown in Fig. 1. To better reflect the changing topology of CR-VANETs, we allow the change of lanes when a vehicle is overtaking the vehicle ahead. We assume all the OBUs accelerate and decelerate with acceleration $a>0$ by probability $p$, and the velocity of any OBU $i \in \mathcal{N}$ is limited by $v_{\text {min }} \leq v_{i}(t) \leq v_{\text {max }}$ at any time. The mobility constraints are listed as follows:

1. The OBUs are randomly placed on both lanes within length $L$ when the simulation begins.

2. The initial speed of OBU $i \in \mathcal{N}$, denoted by $v_{i}(0)$, is randomly given in $\left[v_{\text {min }}, v_{\text {max }}\right]$.

3. The speed of OBU $i \in \mathcal{N}$ satisfies:

$$
v_{i}(t+1)= \begin{cases}v_{i}(t), & 1-2 p \\ \min \left(v_{i}(t)+a, v_{\text {max }}\right), & p \\ \max \left(v_{i}(t)-a, v_{\text {min }}\right), & p\end{cases}
$$

where $p$ is the probability of acceleration or deceleration.

4. For any $\mathrm{OBU} i \in \mathcal{N}$ with $\mathrm{OBU} j_{1}$ ahead in the same lane and $\mathrm{OBU} j_{2}$ ahead in the other lane, OBU $i$ switches to the other lane if $d_{i, j_{1}}(t) \leq d_{s}$ and $d_{i, j_{2}}(t)>d_{s}$, or OBU $i$ decelerates to $v_{i}(t)=v_{j_{1}}(t)$ if $d_{i, j_{k}}(t) \leq d_{s}, k=1,2$.

\subsection{Non-cooperative Spectrum Sensing and Access}

In non-cooperative manner, the OBUs in $\mathcal{N}$ individually sense the PU channels. For any PU channel $k \in \mathcal{K}$ and any $\mathrm{OBU} i \in \mathcal{N}$, we suppose the probability of miss (i.e., probability of missing the detection of the PU) and false alarm (i.e., probability of the false detection of the PU) are denoted by $P_{m}^{i_{k}}$ and $P_{f}^{i_{k}}$, respectively. For simplicity without loss of of generality, we assume the sensing devices in all OBUs have the same performance for any PU channels, which implies $P_{m}^{i_{k}}=$ $P_{m}, P_{f}^{i_{k}}=P_{f}, \forall i \in \mathcal{N}, k \in \mathcal{K}$.

After sensing all the PU channels, OBU $i$ randomly selects a PU channel in $\mathcal{K}_{i}$ to access, as long as $\mathcal{K}_{i} \neq \emptyset$. To avoid potential collisions among OBUs, the carrier sense multiple access with collision avoidance (CSMA/CA) protocol has been adopted by the OBUs broadcasting at the current slot. Each OBU $i \in \mathcal{N}$ can only access the PU channel when no other OBUs are using it. If a data collision is detected by OBU $i, \mathrm{OBU}$ $i$ will stop broadcasting and randomly choose another time point to access.

\section{Cooperative Spectrum Sensing and Access}

For the PCD problem in the considered p2p network, it is natural for the OBUs to cooperate with each other by sharing the sensing result or avoiding potential data collisions. In this section, we introduce the coalition game to model the cooperation of OBUs, in which we suppose it is beneficial for OBUs to contribute for the file delivery process with each successfully delivered 
segment corresponding to unit profit. By proposing a suitable utility function which jointly considers the sensing and access performance, we model the PCD problem as a coalitional game with non-transferable utility.

\subsection{Utility Function}

For any given PU channel $k \in \mathcal{K}$, the OBUs broadcasting at the current slot, the set of which is denoted by $S^{k}$, cooperatively sense the spectrum and simultaneously broadcast at PU channel $k$. Using OR-rule [14] (the PU channel is decided to be empty only when the sensing results of all the SUs are empty), the miss-detection and the false-alarm probabilities of the cooperative spectrum sensing for any OBU in $S^{k}$ are, respectively, given by

$$
Q_{m}^{k}\left(S^{k}\right)=P_{m}\left|S^{k}\right|,
$$

and

$$
Q_{f}^{k}\left(S^{k}\right)=1-\left(1-P_{f}\right)^{\left|S^{k}\right|} \text {. }
$$

We denote by $\mathcal{H}_{1}$ the event that PU channel $k$ is actually occupied by a primary user, and by $\mathcal{H}_{0}$ the complementary event of $\mathcal{H}_{1}$. Also, we denote by $\mathcal{H}_{1}^{\prime}$ the hypothesis that the sensing result shows PU channel $k$ is occupied, and $\mathcal{H}_{0}^{\prime}$ the alternative hypothesis. Thus, the possibility that the empty decision of PU channel $k$ is false can be expressed by:

$$
\begin{aligned}
& P^{k}\left(H_{1} \mid H_{0}^{\prime}\right) \\
= & \frac{P^{k}\left(H_{1}\right) P^{k}\left(H_{0}^{\prime} \mid H_{1}\right)}{P^{k}\left(H_{0}^{\prime}\right)} \\
= & \frac{P^{k}\left(H_{1}\right) P^{k}\left(H_{0}^{\prime} \mid H_{1}\right)}{P^{k}\left(H_{0}^{\prime} \mid H_{0}\right) P^{k}\left(H_{0}\right)+P^{k}\left(H_{0}^{\prime} \mid H_{1}\right) P^{k}\left(H_{1}\right)},
\end{aligned}
$$

and the possibility that the empty decision is correct is given by

$$
P^{k}\left(H_{0} \mid H_{0}^{\prime}\right)=1-P^{k}\left(H_{1} \mid H_{0}^{\prime}\right) .
$$

As we noted, the data traffic of PU channel $k$ is modeled as a Poisson process with parameter $\lambda$. Thus, the possibility of no PU data arriving at PU channel $k$ in the current slot is given by $P^{k}\left(H_{0}\right)=e^{-\lambda}$, and the alternative $P^{k}\left(H_{1}\right)=1-P^{k}\left(H_{0}\right)$. For any given group of OBUs $S^{k}$ broadcasting at the current slot, we directly have $P^{k}\left(H_{0}^{\prime} \mid H_{1}\right)=Q_{m}^{k}(S), P^{k}\left(H_{0}^{\prime} \mid H_{0}\right)=1-Q_{f}^{k}(S)$. Thus, $P^{k}\left(H_{1} \mid H_{0}^{\prime}\right)$ and $P^{k}\left(H_{0} \mid H_{0}^{\prime}\right)$ can be expressed by $P_{m}, P_{f}, \lambda$ and $S^{k}$, where $P^{k}\left(H_{0} \mid H_{0}^{\prime}\right)$ represents the probability of successful channel access and $P^{k}\left(H_{1} \mid H_{0}^{\prime}\right)$ represents the probability of data collision with PUs.

For quantifying the contribution made by each OBU in $S^{k}$ broadcasting at PU channel $k$, we need to determine the only segment broadcasted by each member of $S^{k}$. For maximizing individual profits, we suppose each OBU in $S^{k}$ chooses the "most valuable" segment $x_{i}^{*} \in \mathcal{M}_{i}$ to broadcast, which satisfies

$$
\sum_{j \in \mathcal{N}_{i}^{*}\left(x_{i}^{*}\right)} p_{i, j}^{k} \geq \sum_{j \in \mathcal{N}_{i}^{*}\left(x_{i}\right)} p_{i, j}^{k}, \forall x_{i} \neq x_{i}^{*}, x_{i}, x_{i}^{*} \in \mathcal{M}_{i},
$$

where $\mathcal{N}_{i}^{*}(x)$ represents the set of OBUs in $\mathcal{N}_{i}^{*}$ lacking $x$, which is given by

$$
\mathcal{N}_{i}^{*}(x)=\left\{j \in \mathcal{N}_{i}^{*} \mid x \notin \mathcal{M}_{j}\right\}
$$

As we see, the proposed greedy algorithm for choosing the broadcasted segments selects the segments that can be successfully delivered to the most interested OBUs, which corresponds to the largest profits. As we noted, each successful delivery of one segment to an OBU will bring unit profit to the transmitter. Thus, the expecting profits of OBU $i \in S^{k}$ can be given as

$$
R_{i}\left(S^{k}\right)=\sum_{j \in \mathcal{N}_{i}^{*}\left(x_{i}^{*}\right)} p_{i, j}^{k},
$$

where $x_{i}^{*}$ is the "most valuable" segment for OBU $i$ satisfying (9). Additionally, as the p2p network is also a cognitive radio network, the accuracy of spectrum sensing should also be considered. By introducing the probability that the empty decision is correct in (8), we have the throughput of OBU $i \in S^{k}$ given by

$$
U_{i}\left(S^{k}\right)=P^{k}\left(H_{0} \mid H_{0}^{\prime}\right) R_{i}\left(S^{k}\right) .
$$

As we see, for calculating the value of $U_{i}\left(S^{k}\right)$, certain information needs to be exchanged among the OBUs. This cost in V2V transmissions is necessary and should be considered in the utility function. We consider the following linear cost function with a pricing factor denoted by $\beta$ :

$$
C_{i}\left(S^{k}\right)= \begin{cases}\beta\left|S^{k}\right|, & \text { if }\left|S^{k}\right|>1, \\ 0, & \text { otherwise }\end{cases}
$$

Thus, given the payment formulated in (12) and the cost in (13), the utility function of any OBU $i \in S^{k}$ is given by

$$
V_{i}\left(S^{k}\right)=U_{i}\left(S^{k}\right)-C_{i}\left(S^{k}\right)
$$

This utility function represents the expecting contribution that OBU $i \in S^{k}$ makes in the current slot and equally the payoff OBU $i$ achieves. Based on this utility function, we consider the cooperation and competition among OBUs by coalitional game model in the following subsection.

\subsection{Coalitional Game}

We suppose the group of OBUs $\mathcal{N}^{k} \subset \mathcal{N}$ are competing for the opportunity of broadcasting at PU channel $k$ 
(the rest OBUs, the set of which is $\mathcal{N} \backslash \mathcal{N}^{k}$, have already got the chance of broadcasting at other PU channels). As we noted, for maximizing their individual profits given by (12), the OBUs may choose to form coalitions to compete against each other. For mathematically modeling the competition and cooperation among OBUs, we introduce the coalitional game with nontransferable utility [15-17]:

Definition 1: A coalitional game with non-transferable utility is defined by a pair $(\mathcal{N}, V)$, where $\mathcal{N}$ is the set of players and $V$ is a mapping such that for each coalition of players $S \subseteq \mathcal{N}, V(S)$ is the payoff vector that players in $S$ can achieve.

The concept of non-transferable utility means that each member in a given coalition has a specific profit or performance, and their profits cannot are nontransferable even they are in the same coalition. By adopting the value function in (14) and substituting $S^{k}, \mathcal{N}^{k}$ for $S, \mathcal{N}$, we directly have that the proposed problem can be modeled as a coalitional game with nontransferable utility:

Remark 1: The proposed problem can be modeled as a $\left(\mathcal{N}^{k}, V\right)$ coalitional game with non-transferable utility where the mapping $V$ is given by

$$
\begin{aligned}
V\left(S^{k}\right)=\left(V_{i_{1}}\left(S^{k}\right), V_{i_{2}}\left(S^{k}\right), \ldots, V_{i_{\mid S k} \mid}\left(S^{k}\right)\right), & \\
& i_{l} \in S^{k}, \forall l=1, \ldots,\left|S^{k}\right| .
\end{aligned}
$$

In this coalitional game, the players (the OBUs) can choose to join or leave any coalition based on its own payoff given in (14). If the structure of the players can finally get to a stable situation (no players has the incentive to leave its current coalition), then each coalition in the final structure represents an interest group that competes for the broadcasting opportunity in PU channel $k$. If coalition $S^{k}$ gets the chance of broadcasting in PU channel $k$, then the OBUs in $S^{k}$ simultaneously broadcast their "most valuable" segments in PU channel $k$ at the current slot. To maximize the profits of the entire network, we suppose the broadcasting coalition is the one with the largest total payoff, which is defined as

$$
T\left(S^{k}\right)=\sum_{i \in S^{k}} V_{i}\left(S^{k}\right) .
$$

Remark 2: In the proposed $\left(\mathcal{N}^{k}, V\right)$ coalitional game with non-transferable utility, due to the changing network topology and the benefit-cost tradeoffs from cooperation as expressed in (15), any coalitional structure may form in the network and the grand coalition $\left(\mathcal{N}^{k}\right)$ is seldom beneficial considering the potential interference and collisions. Hence, the proposed game is classified as a coalition formation game with non-transferable utility.

By carefully inspecting the expression of the payoff in (14), we can see the OBUs with no interference to each other always have the incentive to form a coalition, as the transmission rate in (11) keeps the same while the accuracy of sensing in (8) can be improved in cooperative manner. On the other hand, the OBUs close to each other (always means interference from a line of sight) seldom form a coalition because of the severe interference. Thus, the grand coalition $\mathcal{N}^{k}$ is seldom the outcome of the coalitional game due to the potential data collisions. Therefore, the proposed coalitional game is a $\left(\mathcal{N}^{k}, V\right)$ coalition formation game [17]. We will devise a coalition formation algorithm to get these disjoint coalitions in the next section.

\section{Coalition Formation Algorithm}

Given the above analysis, the cooperative manner of OBUs for a single PU channel can be classified as a coalition formation game with non-transferable utility, where the OBUs form several disjoint coalitions to maximize their own payoffs. In this section, we devise a distributed coalition formation algorithm and additionally propose the entire approach for the PCD problem.

\subsection{Coalition Formation Concepts}

Before constructing a coalition formation algorithm, we first introduce some necessary concepts, taken from [18]. Here, the set of players is denoted by $\mathcal{N}^{k}$ representing the OBUs cooperatively competing for PU channel $k$. First, we give the concept of coalition partition for describing the structure of players.

Definition 2: A coalition partition is defined as a set of disjoint coalitions that cover the player set, i.e., for any coalitional partition $\Pi^{k}=\left\{S_{1}^{k}, \ldots, S_{r}^{k}\right\}$ we have $\forall l, S_{l}^{k} \subseteq$ $\mathcal{N}^{k}$ and $\bigcup_{l=1}^{r}\left\{S_{l}^{k}\right\}=\mathcal{N}^{k}$. Also, we denote by $S_{\Pi^{k}}^{k}(i)$, the coalition $S_{l}^{k} \in \Pi^{k}$ including OBU $i$, i.e., $i \in S_{l}^{k}$.

One key approach in coalition formation is to enable the players to join or leave a coalition based on welldefined preferences. Therefore, we need to introduce the concept of preference relation, using which the players can decide which coalition they prefer more to be a member of.

Definition 3: For any player $i \in \mathcal{N}^{k}$, a preference relation or order $\geq_{i}$ is defined as a complete, reflexive, and transitive binary relation over the set of all coalitions that player $i$ can possibly form, i.e., the set $\left\{S_{l}^{k} \subseteq \mathcal{N}: i \in S_{l}^{k}\right\}$.

For any given player $i \in \mathcal{N}^{k}, S_{1}^{k} \geq_{i} S_{2}^{k}$ implies that player $i$ prefers being a member of coalition $S_{1}^{k}$ with $i \in S_{1}^{k}$ over being a member of coalition $S_{2}^{k}$ with $i \in S_{2}^{k}$, or at least, $\mathrm{OBU} i$ prefers both coalitions equally. In this paper, the preference of any OBU $i \in \mathcal{N}^{k}$ with $i \in S_{1}^{k}, S_{2}^{k}$ is quantified as follows:

$$
S_{1}^{k} \geq_{i} S_{2}^{k} \Leftrightarrow V_{i}\left(S_{1}^{k}\right) \geq V_{i}\left(S_{2}^{k}\right) \& S_{1}^{k} \in A^{k}(i),
$$


where $A^{k}(i)$ is the set of OBU $i$ 's "friendly" coalitions defined by

$$
\begin{aligned}
A^{k}(i)=\left\{S_{l}^{k} \subseteq \mathcal{N}^{k}\right. & \mid i \in S_{l}^{k} \& \\
& \left.\forall j \in S_{l}^{k} \backslash\{i\}, V_{j}\left(S_{l}^{k}\right) \geq V_{j}\left(S_{l}^{k} \backslash\{i\}\right)\right\} .
\end{aligned}
$$

As we see, a coalition $S_{l}^{k}$ is "friendly" to OBU $i$, only when OBU $i$ 's joining increases or at least maintains the payoffs of all the other OBUs in $S_{l}^{k}$. Thus, the definition of preference implies that OBU $i$ prefers being a member of $S_{1}^{k}$ over $S_{2}^{k}$ only when OBU $i$ gains an increase in individual profit and meanwhile no other OBUs in $S_{1}^{k}$ suffers a decrease because of OBU $i$ 's joining. The asymmetric counterpart of $\geq_{i}$, denoted by $>_{i}$, is defined as

$$
S_{1}^{k}>_{i} S_{2}^{k} \Leftrightarrow V_{i}\left(S_{1}^{k}\right)>V_{i}\left(S_{2}^{k}\right) \& S_{1}^{k} \in A^{k}(i) .
$$

Based on the defined preference relation, the basic operation in our proposed coalition formation algorithm, the switch operation, is defined as follows:

Definition 6: Given a partition $\Pi^{k}=\left\{S_{1}^{k}, \ldots, S_{r}^{k}\right\}$ of the players set $\mathcal{N}^{k}$, if player $i \in \mathcal{N}^{k}$ performs a switch operation from $S_{\Pi^{k}}^{k}(i)=S_{m}^{k}$ to $S_{l}^{k} \in \Pi^{k} \cup\{\emptyset\}, S_{l}^{k} \neq S_{\Pi^{k}}^{k}(i)$, then the current partition $\Pi^{k}$ of $\mathcal{N}^{k}$ is modified into a new partition $\Pi^{k^{\prime}}$ such that $\Pi^{k^{\prime}}=\left(\Pi^{k} \backslash\left\{S_{m}^{k}, S_{l}^{k}\right\}\right) \cup$ $\left\{S_{m}^{k} \backslash\{i\}, S_{l}^{k} \cup\{i\}\right\}$.

Definition 7: Given any player $i \in \mathcal{N}^{k}$, the history collection $H^{k}(i)$ is defined as the set of coalitions that player $i$ visited and then left for the competition of PU channel $k$.

\subsection{Distributed Coalition Formation Algorithm}

For competing a given PU channel $k$, we propose a coalition formation algorithm that allows the OBUs in $\mathcal{N}^{k}$ to make distributed decisions as to which coalitions they decide to join at any time slot. The basic rule of the algorithm is as follows:

Basic Rule: Given a partition $\Pi^{k}=\left\{S_{1}^{k}, \ldots, S_{r}^{k}\right\}$ of the OBUs set $\mathcal{N}^{k}$, a switch operation from $S_{\Pi^{k}}^{k}(i)$ to $S_{l}^{k} \in$ $\Pi^{k} \cup\{\emptyset\}, S_{l}^{k} \neq S_{\Pi^{k}}^{k}(i)$ is allowed for any OBU $i \in \mathcal{N}^{k}$, if and only if $S_{l}^{k} \cup\left\{i>_{i} S_{\Pi^{k}}^{k}(i)\right.$ and $S_{l}^{k} \cup\{i\} \notin H^{k}(i)$.

For any partition $\Pi^{k}$, the basic rule provides a mechanism whereby any OBU can leave its current coalition $S_{m}^{k}$ and join another coalition $S_{l}^{k} \in \Pi^{k}$, given that the new coalition is strictly preferred over $S_{m}^{k}$ through the preference relation defined by (19). Thus, the basic rule can be seen as an individual rule abided by each member of $\mathcal{N}^{k}$, to move from its current coalition to a new coalition for improving its payoff, meanwhile maintains the profits of other members of this new coalition. Further, we suppose whenever an OBU decides to switch from its current coalition $S_{m}^{k} \in$ $\Pi^{k}$ to join a different coalition, coalition $S_{m}^{k}$ is stored
Table 1. The Coalition Formation Algorithm for On-board Units Competing for Primary User Channel $k$

Given any partition $\Pi_{\text {initial }}^{k}$ of the OBUs set $\mathcal{N}^{k}$
with the initialized history collections $H^{k}(i)=$
$\emptyset, \forall i \in \mathcal{N}^{k}$, the OBUs engage in the coalition
formation algorithm as follows:
* repeat
For a randomly chosen OBU $i \in \mathcal{N}^{k}$, with current partition $\Pi_{\text {current }}^{k}\left(\Pi_{\text {current }}^{k}=\Pi_{\text {initial }}^{k}\right.$ in the first round)

1. OBU $i$ searches for a possible switch operation according to the basic rule in Section IV.

2. If such a switch exists, OBU $i$ performs the following steps:

(a) Updates the history collection $H^{k}(i)$ by adding coalition $S_{\Pi_{\text {current }}^{k}}^{k}(i)$ before leaving it.

(b) Leaves coalition $S_{\Pi_{\text {current }}^{k}}^{k}(i)$.

(c) Joins the new coalition $S_{\Pi_{\text {next }}^{k}}^{k}(i)$ that improves its payoff.

* until the partition converges to a final Nashstable partition $\Pi_{\text {final }}^{k}$.

in its history collection $H^{k}(i)$ (if $\left|S_{m}^{k}\right|>1$ ). With the order in which the OBUs make their switch operations considered to be random, the complete form of our distributed coalition formation algorithm is shown in Table 1.

\subsection{Convergence and Stability}

The convergence of the proposed coalition formation algorithm is guaranteed as follows:

Proposition 1: Starting from any initial coalitional partition $\Pi^{k}{ }_{\text {initial }}$, the proposed algorithm maps to a sequence of switch operations, which always converges to a final partition $\Pi^{k}$ final composed of a number of disjoint coalitions.

Proof: Denote by $\Pi_{n, i}^{k}$ the partition formed at the time OBU $i \in \mathcal{N}^{k}$ needs to act after the $n$ switch operations made by one or more OBUs. Given any initial starting partition $\Pi_{\text {initial }}^{k}=\Pi_{0, i}^{k}$, the coalition formation algorithm consists of a sequence of switch operations, e.g., $\Pi_{0,1}^{k} \rightarrow \Pi_{1,3}^{k} \rightarrow \ldots \rightarrow \Pi_{n, i}^{k} \ldots \rightarrow \Pi_{m, j}^{k} \ldots$. For any two partition $\Pi_{n, i}^{k}$ and $\Pi_{m, j}^{k}$, such that $n<m, \Pi_{m, j}^{k}$ is a result of the transformation of $\Pi_{n, i}^{k}$ after $m-n$ switch operations, we have have $\Pi_{n, i}^{k} \neq \Pi_{m, j}^{k}$ since each 
OBU maintains a history collection to avoid joining a coalition repeatedly. As the number of partitions of a set is finite (given by the Bell number [19]), then the number of transformations in the sequence is finite. Hence, the algorithm will always terminate after finite iterations and converge to a final partition $\Pi_{\text {final }}^{k}$.

The stability of the final partition $\Pi_{\text {final }}^{k}$ resulting from the proposed algorithm can be studied using the following concept from the hedonic games [18].

Definition 8: A partition $\Pi^{k}=\left\{S_{1}^{k}, \ldots, S_{r}^{k}\right\}$ is Nashstable, if $\forall i \in \mathcal{N}^{k}, S_{\Pi^{k}}^{k}(i) \geq_{i} S_{l}^{k} \cup\{i\}$ for all $S_{l}^{k} \in \Pi^{k} \cup\{\emptyset\}$.

The definition of Nash-stable implies that any coalition partition $\Pi^{k}$ in which no OBU has an incentive to move from its current coalition to another coalition in $\Pi^{k}$ or to deviate and act alone, is considered to be Nash-stable.

Proposition 2: Any final partition $\Pi_{\text {final }}^{k}$ resulting from the proposed coalition formation algorithm is Nash-stable.

Proof: Suppose the final partition $\Pi_{\text {final }}^{k}$ is not Nashstable, which implies there exists an OBU $i \in \mathcal{N}^{k}$, and a coalition $S_{l}^{k} \in \Pi_{\text {final }}^{k}$ such that $S_{l}^{k} \cup\{i\}>_{i} S_{\Pi_{\text {final }}^{k}}^{k}(i)$. Based on the basic rule, OBU $i$ can perform a switch operation from $S_{\Pi_{\text {final }}^{k}}^{k}(i)$ to $S_{l}^{k}$, which contradicts the fact that $\Pi_{\text {final }}^{k}$ is the final partition. Thus, any final partition $\Pi_{\text {final }}^{k}$ resulting from the proposed coalition formation algorithm is Nash-stable.

\subsection{Cooperative Approach for Popular Content Distribution}

The competition for PU channel $k$ among OBUs $\mathcal{N}^{k}$ has been modeled as a coalition formation game with non-transferable utility and a convergent coalition formation algorithm with a Nash-stable outcome has been given in Table 1. Consider the considered scenario has multiple PU channels, we propose a sequential method for allocating the OBUs in $\mathcal{N}$ to the PU channels in $\mathcal{K}$. To be specific, the OBUs in $\mathcal{N}$ implement the coalition formation algorithm on each channel sequentially. After the calculation for a PU channel, the OBUs achieving the opportunity of broadcasting will quit the competition for the rest PU channels.

A summary of the entire approach for the proposed PCD problem is shown in Table 2. In the first stage, a distributed neighbor discovery method, e.g., those in $[20,21]$, is used to capture any changes in the network structure and record the necessary information for the following calculation. In the second stage, the sequential coalition formation algorithm has been used to allocate PU channels for the OBUs in the network. In the thrid stage, the OBUs access their corresponding PU channels by broadcasting their most valuable segments.
Table 2. The Approach for the Popular Content Distribution Problem

With the initial segments randomly distributed among $\mathcal{M}$, the OBUs periodically perform the following three stages:

Stage I: Network Discovery

Each OBU performs a neighbor discovery algorithm to obtain the necessary information for calculating individual payoff in (14), and the initial partition is set as $\Pi_{\text {initial }}=\mathcal{N}$.

\section{Stage II: Sequential Coalition Formation}

For PU channel $k=1,2, \ldots,|\mathcal{K}|$, we performs the following steps:

1. Determine the OBUs competing for the current PU channel $\mathcal{N}^{k}=\mathcal{N}^{k-1} \backslash S^{k-1}$ (For PU channel $1, \mathcal{N}^{1}=\mathcal{N}$ ) where $S^{k-1}$ is the broadcasting coalition for PU channel $k-1$.

2. The OBUs in $\mathcal{N}^{k}$ perform the coalition formation algorithm in Table 1 to obtain the final partition $\Pi_{\text {final }}^{k}$.

3. The coalition with the largest total payoff (16) is decided to be the broadcasting coalition $S^{k}$.

\section{Stage III: Spectrum Sensing and Broadcasting \\ For any broadcasting coalition $S^{k}$ in PU channel $k$, the OBUs in $S^{k}$ cooperatively sense PU channel $k$ with OR-rule. If the sensing result is empty, the OBUs in $S^{k}$ simultaneously access PU channel $k$ by broadcasting their "most valuable" segments defined in (9).}

To adapt to the changing network topology, the three stages are repeated periodically.

In the non-cooperative manner, each OBU has to sense all the PU channels (or at least enough PU channels to fine an empty one) before deciding which to access. In the proposed approach, the channel access decision is based on the expecting data throughput which needs no sensing result from any OBU. Thus, the potential PU channel for any OBU can be decided before any spectrum sensing. Consequently, in our proposed approach, each OBU only needs to sense the PU channel that has been allocated to it rather than sense the entire spectrum, which decreases the sensing cost or equally increases the transmission time. 
Table 3. Parameters for Simulation

\begin{tabular}{|l|l|}
\hline$N=5 \sim 15$ & number of OBUs in the network \\
\hline$L=500 \mathrm{~m}$ & initial length of the fleet of vehicles \\
\hline$M=5 \sim 15$ & $\begin{array}{l}\text { number of segments of the entire } \\
\text { file }\end{array}$ \\
\hline$K=1 \sim 2$ & number of PU channels \\
\hline$M c=1 \mathrm{Mbit}$ & the size of the entire popular file \\
\hline$v_{\min }=20 \mathrm{~m} / \mathrm{s}$ & the minimal speed \\
\hline$v_{\max }=40 \mathrm{~m} / \mathrm{s}$ & the maximal speed \\
\hline$d_{s}=100 \mathrm{~m}$ & the safe distance \\
\hline$a=2 \mathrm{~m} / \mathrm{s}^{2}$ & the acceleration \\
\hline$p=0.1$ & $\begin{array}{l}\text { the probability for acceleration or } \\
\text { deceleration }\end{array}$ \\
\hline$\rho_{c}=0.3 \sim 0.6$ & the initial content density \\
\hline$\beta=0.01$ & the pricing factor \\
\hline$n=4$ & the exponent for pathloss \\
\hline$W=30 \mathrm{MHz}$ & the bandwidth \\
\hline$\alpha=10^{6}$ & $\begin{array}{l}\text { the signal-to-noise rate at the trans- } \\
\text { mitter }\end{array}$ \\
\hline$\lambda=0.1 \sim 0.3$ & $\begin{array}{l}\text { the arriving rate of PU traffic per } \\
\text { slot }\end{array}$ \\
\hline$P_{m}=0.1$ & the possibility of missing \\
\hline$P_{f}=0.1$ & the possibility of false alarm \\
\hline
\end{tabular}

\section{Simulation Results}

In this section, the performance of the proposed approach in Table 2 is simulated in various environmental conditions and compared with the noncooperative manner in many aspects. The parameters are taken from a general highway scenario as shown in Table 3. Here, the content density $\rho_{c}$ is defined as the average number of initially possessed segments dividing the total number of segments $M$, which represents the level of initial content dissemination. Also, we assume each slot lasts 1 second.

In Fig. 2, we show the number of total possessed segments, given by $P(t)=\sum_{i \in \mathcal{N}}\left|\mathcal{M}_{i}\right|$, for networks with $N=5, M=5, K=1,2 \rho_{c}=0.6, \lambda=0.1$, where the vertical coordinate has been normalized by the total demand NM. The performance of the proposed algorithm is compared with the non-cooperative scheme in both conditions of single PU channel and multiple PU channels. In the non-cooperative scheme, each OBU individually senses the spectrum and makes an individual decision on the presence or absence of the PU for each PU channel. Then, each OBU randomly accesses one of the vacant channels where no neighbor OBUs are broadcasting. Fig. 2 shows that, as the transmitting time increases, the number of total possessed increases for both the methods. However, our proposed algorithm achieves a better performance in both the total number and the ascending rate. In the non-cooperative manner, the OBUs sense the

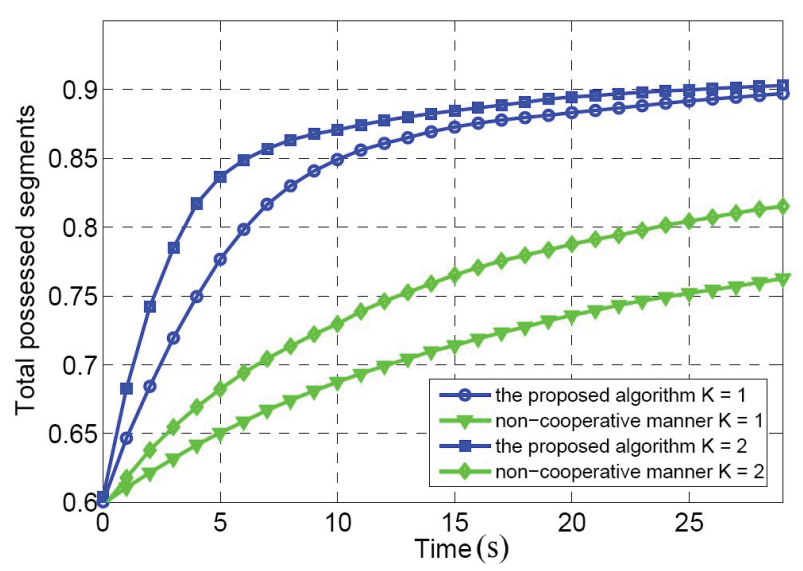

Figure 2. Total segments of all OBUs by the proposed approach and the non-cooperative manner as a function of time for networks with $N=5, M=5, K=1,2, \rho_{c}=0.6, \lambda=0.1$.

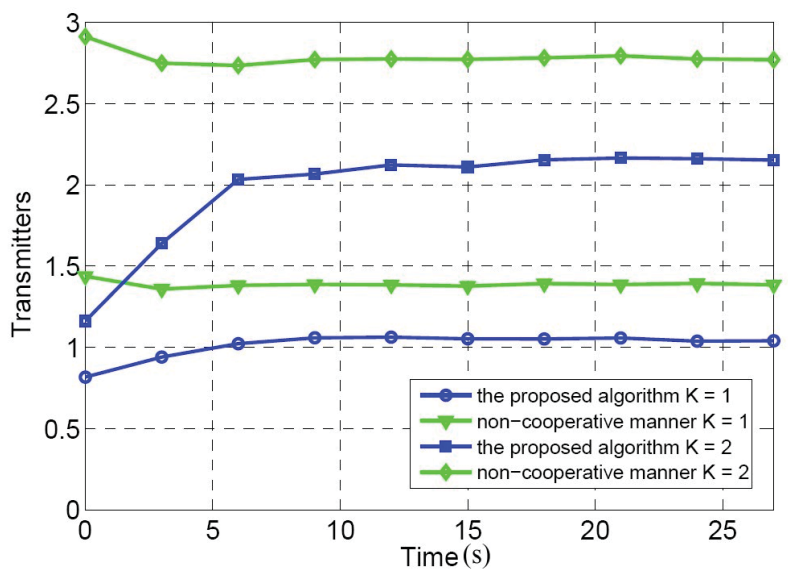

Figure 3. Total transmitters in the network by the proposed approach and the non-cooperative manner as a function of time for networks with $N=5, M=5, K=1,2, \rho_{c}=0.6, \lambda=0.1$.

spectrum individually and access by avoiding potential collisions (though still with hidden terminal problem). However, in our proposed algorithm, the spectrum sensing and channel access have been jointly in the value function of the coalition formation game, which represents the expecting throughput for each OBU. Aiming at maximizing the throughput rather than simply avoiding collisions makes our algorithm more efficient than the non-cooperative manner.

In Fig. 3, we show the number of transmitters for the networks with $N=5, M=5, K=1,2 \rho_{c}=0.6, \lambda=$ 0.1 . As we can see, the number of transmitters of the proposed approach is lower than the non-cooperative manner in both single PU channels or multiple PU channels. In the non-cooperative manner, if OBU $i$ detects that no other OBUs are using an empty PU channel, OBU $i$ immediately accesses the spectrum 


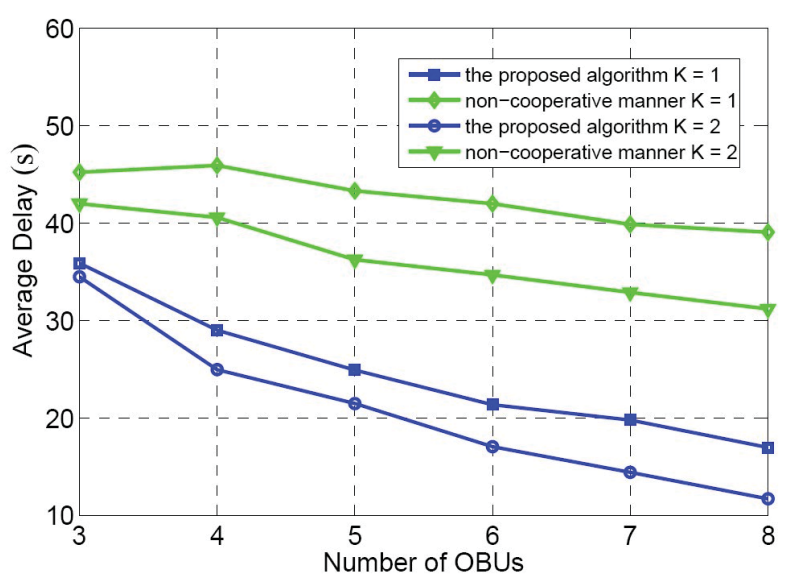

Figure 4. Average delay by the proposed approach and the non-cooperative manner as a function of $N$ for networks with $M=5, K=1,2, \rho_{c}=0.6, \lambda=0.1$.

without considering how many OBUs could actually receive the broadcasting segment. However, in our proposed approach, the channel conditions together with the wanted segments of each OBUs are considered, which highly reduces unnecessary broadcastings when the transmitter is far way from other OBUs, or the segment for broadcasting is not requested by neighborhood OBUs. Fig. 3 shows that, although we do not consider any energy model in our scenario, the proposed approach still achieves a low power consumption.

In Fig. 4, we show the average delay experienced by the OBU for achieving the entire file as a function of $N$ for networks with $M=5, K=1,2 \rho_{c}=0.6, \lambda=0.1$. In the proposed PCD problem, the core parameter considered by the content owner is the average delay experienced by the OBUs, which is generally defined as $\tau_{a}=\tau_{t} / N$ with $\tau_{t}$ representing the total delay experienced by all OBUs. For any given content distribution scheme, $\tau_{t}$ is given by the area between the cumulative demand curve and cumulative service curve [22]. In our scenario, we assume all arrivals of demand occur instantaneously [23] at the beginning of $\mathrm{p} 2 \mathrm{p}$ transmission and stay unchanged ever since. Thus, the demand curve is a constant NM and the average delay $\tau_{a}$ is given by

$$
\tau_{a}=\frac{1}{N} \int_{t=0}^{\tau_{m}}[N M-P(t)] d t,
$$

where $P(t)$ is the cumulative service curve representing the number of segments possessed by the OBUs, and $\tau_{m}$ is the maximal delay defined by $P\left(\tau_{m}\right)=$ $N M$ when the PCD completes. The performance of the proposed algorithm is compared with the noncooperative manner in conditions of single PU channel

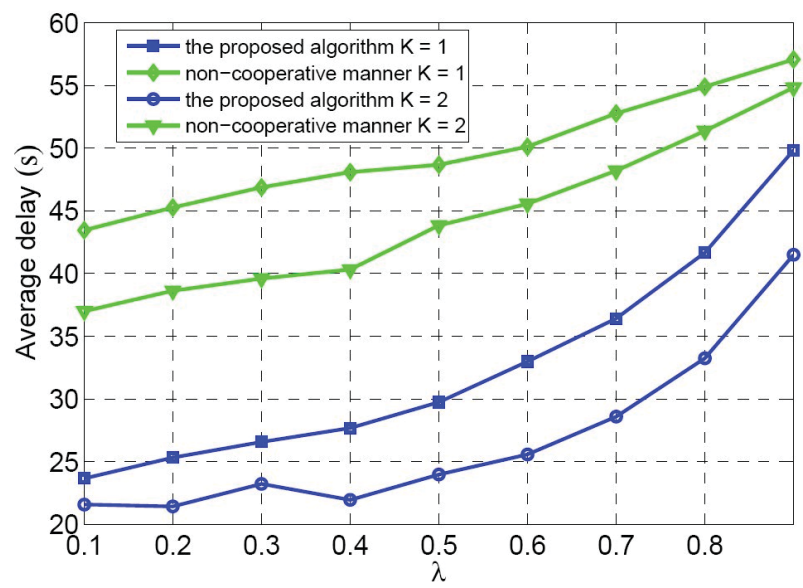

Figure 5. Average delay by the proposed approach and the non-cooperative manner as a function of $\lambda$ for networks with $N=5, M=5, K=1,2, \rho_{c}=0.6$.

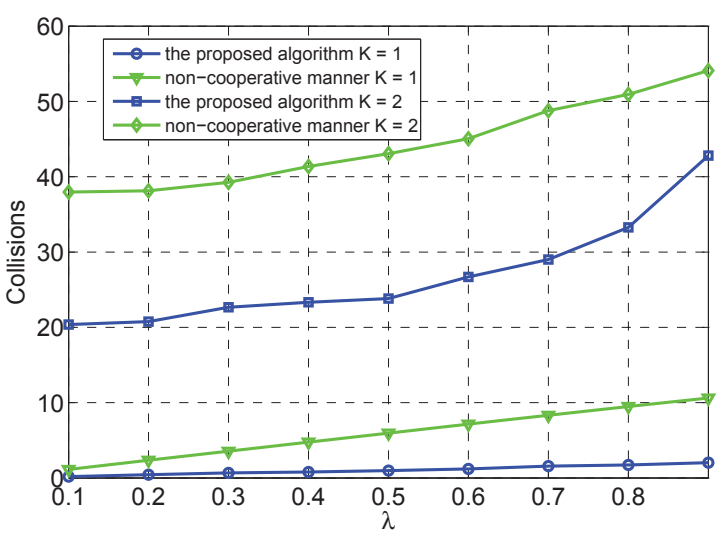

Figure 6. Collisions with primary users by the proposed approach and the non-cooperative manner as a function of $\lambda$ for networks with $N=5, M=5, K=1,2$.

and multiple PU channels. Fig. 4 shows our proposed method enjoys an average delay approximately $40 \%$ smaller than the non-cooperative scheme. As we see, the average delay decreases as the number of OBUs increases by both methods, which can be explained by the increasing connectivity among OBUs.

In Fig. 5, we show the average delay as a function of $\lambda$ for networks with $N=5, M=5, K=1,2, \rho_{c}=0.6$. The performance of the proposed algorithm is compared with the non-cooperative manner in conditions of single PU channel and multiple PU channels. As the arriving rate of PU data $\lambda$ increases, the potential channels and time slots for SUs (the OBUs) decrease, which increases the average delay of the PCD problem. Also, Fig. 5 shows that our method has a better performance than the non-cooperative manner with $40 \%$ decrease in average delay for any level of PU traffic. 
In Fig. 6, we show the collisions with PU traffic as a function of $\lambda$ for networks with $N=5, M=$ $5, K=1,2, \rho_{c}=0.6$. The performance of the proposed algorithm is compared with the non-cooperative manner in conditions of single PU channel and multiple PU channels. As the $\lambda$ increases, the PU traffic becomes heavy and the probability of collisions also increases as shown in Fig. 6. As we see, our proposed algorithm has less collisions compared with the non-cooperative manner, which means our proposed approach has a smaller interference to the PUs.

\section{Conclusions}

In this paper, we have proposed a p2p scheme using V2V communications to solve the PCD problem in VANETs. Cognitive radio technique has been introduced for V2V communications which makes the network a CR-VANET. By carefully defining the utility function, we have proposed a coalition formation game with non-transferable utility for modeling the behavior of the OBUs. Further, we have introduced a coalition formation algorithm which could map into a sequence of switch operations and converge to a final Nash-stable partition. Based on the algorithm, we have proposed the overall approach for the PCD problem. In the simulation part, we have shown that our approach has a better performance with various conditions in average delay, power consumption, and interference to PUs, compared with the non-cooperative scheme.

\section{References}

[1] Olariu, S. and Weigle, M. A. C. [eds.] (2009) Vehicular Networks: From Theory to Practice. (London, UK: Chapman \& Hall/CRC).

[2] Hartenstein, H. and Laberteaux, K. P. (2008) A tutorial survey on vehicular ad hoc networks. IEEE Communications Magazine 46(6): 164-171.

[3] LI, M., YANG, Z. and Lou, W. (2011) Codeon: cooperative popular content distribution for vehicular networks using symbol level network coding. IEEE Journal on Selected Areas in Communications 29(1): 223-235.

[4] Wang, T. Song, L. and Han, Z. (2013) Coalitional Graph Games for Popular Content Distribution in Cognitive Radio VANETs. IEEE Transactions on Vehicular Technology 31(9): 538-547.

[5] Wang, T. Song, L. Han, Z. and Jiao, B. (2013) Dynamic Popular Content Distribution in Vehicular Networks using Coalition Formation Games. IEEE Journal on Selected Areas in Communications 62(8): 4010-4019.

[6] Nandan, A. Das, S. Pau, G. Gerla M. and Sanadidi, M. Y. (2005) Co-operative downloading in vehicular ad-hoc wireless networks. In Second Annual Conference on Wireless On-demand Network Systems and Services(St. Moritz, Switzerland: IEEE), 32-41.

[7] Fiore, M. and Barcelo-Ordinas, J. M. (2009) Cooperative download in urban vehicular networks. In IEEE 6th International Conference on Mobile Adhoc and Sensor Systems (IEEE), 20-29.

[8] Lua, K. Crowcroft, J. Pias, M. Sharma, R. and Lim, S. (2005) A survey and comparison of peer-to-peer overlay network schemes. IEEE Communications Surveys \& Tutorials 7(2): 72-93.

[9] Saad, W. Han, Z. Huorungnes, A. Niyato, D. and HosSAIN, E. (2011) Coalition formation games for distributed cooperation among roadside units in vehicular networks. IEEE Journal on Selected Areas in Communications 29(1): 48-60.

[10] SaAd, W. Han, Z. Zheng, R. Hjorungnes, A. Basr, T. and Vincent Poor, H. (2012) Coalitional games in partition form for joint spectrum sensing and access in cognitive radio networks. IEEE Journal on Selected Areas in Communications 6(2): 195-209.

[11] Zhang, R. Song, L. Han, Z. and JiaO, B. (2011) Distributed coalition formation of relay and friendly jammers for secure cooperative networks. In IEEE International Conference on Communications (Kyoto, Japan: IEEE), 1-6.

[12] Mecklenbrauker, C. F. Molisch, A. F. Karedal, J. Tufvesson, F. Paier, A. Bernado, L. Zemen, T. Klemp, O. and CzINK, N. (2011) Vehicular channel characterization and its implications for wireless system design and performance. Proc. the IEEE 99(7): 1189-1212.

[13] Mahajan, A. Potnis, N. Gopalan, K. and Wang, A. I. A. (2006) Urban Mobility Models for VANETs. In Proceedings of the 2nd IEEE International Workshop on Next Generation Wireless Networks (IEEE).

[14] Peh, E. and Liang, Y.-C. (2007) Optimization for cooperative sensing in cognitive radio networks. In Proceedings of IEEE Wireless Communication and Networking Conference (Hong Kong, China: IEEE), 27-32.

[15] Myerson, R. B. (1991) Game Theory, Analysis of Conflict (Cambridge, MA, USA: Harvard University Press).

[16] Owen, G. (1995) Game theory (London, UK: Academic Press), 3rd ed.

[17] SaAd, W. Han, Z. Debbah, M. Hjoungnes, A. and Basar, T. (2009) Coalition game theory for communication networks: a tutorial. IEEE Signal Processing Magzine, Special Issue on Game Theory 26(5): 77-97.

[18] Bogomonlaia, A. and Jackson, M. (2002) The stability of hedonic coalition structures. Games and Economic Behavior 38: 201-230.

[19] Ray, D. (2007) A Game-Theoretic Perspective on Coalition Formation (New York, USA: Oxford University Press).

[20] Damljanovic, Z. (2008) Cognitive radio access discovery strategies. In International Symposium on Communication Systems, Networks, and Digital Signal Processing (IEEE).

[21] Arachchige, C. J. L. Venkatesan, S. Mittal, N. (2008) An asynchronous neighbor discovery algorithm for cognitive radio networks. In IEEE Symposium on New Frontiers in Dynamic Spectrum Access Networks (IEEE).

[22] Bertsekas, D. and Gallager, R. [eds.] (1987) Data Networks (Boston, MA: Longman Higher Education).

[23] Qiu, D. and Srikant, R. (2004) Modeling and performance analysis of bittorrent-like peer-to-peer networks. In Proc. ACM SIGCOMM (Karlsruhe, Germany), 367-378. 